\title{
Aspectos ideológicos, gramaticales y léxicos del sexismo lingüístico
}

\author{
Ideological, grammatical and lexical aspects of linguistic sexism
}

\author{
María del Carmen Cabeza Pereiro, ${ }^{1}$ Susana Rodríguez Barcia ${ }^{2}$ \\ ${ }^{1}$ Universidade de Vigo, Facultad de Filología y Traducción, Departamento de Traducción y Lingüística, \\ Vigo, España. Correo electrónico: cabeza@uvigo.es \\ ${ }^{2}$ Universidade de Vigo, Facultad de Filología y Traducción, Departamento de Lengua Española, Vigo, \\ España. Correo electrónico: barcia77@uvigo.es
}

El informe de la RAE firmado por Ignacio Bosque aviva la polémica sobre las intervenciones que pretenden evitar la ocultación de la mujer en los usos lingüísticos. En este artículo nos proponemos una tarea de doble alcance: por una parte, revisar la naturaleza del género gramatical en español desde la perspectiva de la Lingüística Cognitiva. Mostraremos que, en contra de lo que con frecuencia se defiende, sí se deriva una interpretación masculina "por excelencia” de algunos usos pretendidamente genéricos del masculino. Por otro lado, algunas pesquisas en los artículos del diccionario usual de la RAE nos permitirán valorar que todavía perviven fórmulas de minimización de la mujer en esta obra lexicográfica. Esta resistencia a los cambios fundamentados en la igualdad pone de relieve hasta qué punto es necesario mantener viva la reflexión sobre las ideologías en el discurso lingüístico académico.

Palabras clave: género, sexismo lingüístico, ideología, Real Academia Española.

The report of the Real Academia Española signed by Ignacio Bosque has intensified the debate about the recent attempts to avoid women's invisibility in language. In this paper our aim is a double task: on the one hand, we want to analyze the nature of grammatical gender in Spanish from the point of view of Cognitive Linguistics. We will show that, contrary to what has been frequently defended, the allegedly generic use of masculine grammatical gender is actually due to a masculine interpretation of reality. On the other hand, an analysis of some RAE usual dictionary entries will allow us to conclude that some expressions that undervalue women are still remaining in this lexicographic work. This resistance to changes favoring gender equality highlights to what extent it is necessary to keep alive a reflection on ideology in the academic linguistic discourse.

Key words: gender, linguistic sexism, ideology, Real Academia Española. 


\section{INTRODUCCIÓN}

Desde hace poco más de una década, un significativo grupo de hablantes dentro del conjunto de la sociedad panhispánica viene reclamando - fundamentalmente a través de la publicación de guías y manuales con recomendaciones específicas - una intervención en la lengua española que evite los usos sexistas y, en general, que posibilite lo que se ha dado en denominar visibilidad de la mujer, en especial en los ámbitos de la administración. Desde luego, este tema en el que se entremezclan aspectos lingüísticos con aspectos ideológicos se ha puesto de relieve sobre todo tras la publicación del informe "Sexismo lingüístico y visibilidad de la mujer", llevado a cabo por Ignacio Bosque con el respaldo de la RAE (Bosque 2012).

Nuestra perspectiva se basa en la creencia de que la conformación de la lengua depende muy directamente del uso y, por tanto, de los aspectos sociales e ideológicos. Asimismo, en este artículo entendemos la noción de sexismo lingüístico como distribución inicua de prácticas lingüísticas a partir de la preeminencia de un género gramatical con base en motivaciones ideológicas de índole cultural y tradicional. Admitiendo esto, sí sería posible entender la intervención directa sobre diversos usos de la lengua, que iría encaminada hacia la consecución de equidad. Por otra parte, Moreno Cabrera (2012: 4) indica también que habría que tener en cuenta la distinción entre lengua natural y lengua cultivada con el fin de evitar problemas metodológicos y científicos a la hora de entender el tema que constituye nuestro objeto de estudio. Por lenguas cultivadas (Moreno Cabrera 2011, 2012) entenderá aquellas que surgen de la manipulación e intervención intencionada y cultural de las lenguas naturales. En este sentido, no cabe duda de que nuestra problemática cambia de punto de partida. Hemos de entender, pues, que las intervenciones sobre la lengua para evitar usos que algunos hablantes de español consideran discriminatorios u ocultadores de la mujer darían como producto una lengua cultivada (Moreno Cabrera 2011, 2012) en la que la intervención sería no solo lícita, sino necesaria.

En definitiva, es nuestra intención abordar el tema del sexismo lingüístico en el español desde una perspectiva lingüística, pero teniendo en cuenta que los aspectos sociales, culturales e ideológicos no son, ni deben ser, ajenos a las intervenciones sobre la lengua en ámbitos específicos (apartado 2). La perspectiva cognitivista (Langacker 1987, 2000; Lakoff 1987) nos ofrecerá el punto de partida para fundamentar la base semántica que subyace a la distinción de género en una parte de los nombres de la lengua española (apartado 3). En el apartado 4 revisaremos los conceptos de asignación de género y resolución de género en las lenguas, basándonos en Corbett (1991) y en el 5 abordaremos los aspectos gramaticales del sexismo lingüístico en la lengua española, centrándonos especialmente en el análisis de los llamados usos genéricos del masculino. Asimismo, también nos interesa poner el acento en el papel de la Real Academia Española como organismo rector de norma gramatical y léxica (apartado 6) en una polémica en la que, como ha de ser, la última palabra la tendrán los hablantes, hombres y mujeres.

\section{LENGUA E IDEOLOGÍA}

La lengua no es un sistema ajeno a la cultura en la que se desarrolla; lejos de eso, podría entenderse como el resultado de fundir el código con la adaptación biológica y con el contexto histórico-social. Por lo tanto, se trata de un producto 
cultural con un marcado carácter simbólico para sus hablantes. En este sentido, es necesario introducir la noción de modelos culturales (Cole 1999: 121) pues, en el ámbito de los hispanohablantes que nos ocupa en esta ocasión, los modelos culturales que sustentan los roles tradicionales atribuidos a la mujer y al hombre subyacen sin duda bajo las diferentes posturas que se mantienen en relación con las intervenciones de orientación no sexista en los trabajos de carácter lingüístico y, en definitiva, en las distintas producciones discursivas. Los modelos culturales serán, como defiende Cole "esquemas culturales compartidos intersubjetivamente", por lo que en ellos podrá identificarse un vehículo firme pero sutil de consolidación ideológica. Asimismo, como defiende van Dijk (2010: 17) "las ideologías pueden ser vistas como la base de la auto-imagen" y, además, "las ideologías controlan más específicamente las actitudes compartidas por grupos"; por lo que, en el caso del sexismo lingüístico, no resulta en absoluto baladí el análisis de las ideologías en este ámbito en el que la imagen de la mujer y las actuaciones sociales relacionadas con ella cobran vital importancia para el desarrollo sano y satisfactorio de la sociedad panhispánica.

Por otra parte, y en cuanto a la difusión y mantenimiento de los modelos culturales, ya no parece discutible concebir el desarrollo personal como una construcción cultural conseguida a partir de interacciones sociales conjuntas en las que la educación resulta fundamental (Vygotsky 1995), y de esta realidad es posible dirimir que el entorno familiar y la escuela son esenciales en las etapas iniciales de la formación de cada individuo. La lengua, como vehículo de comunicación, participa activamente en la empresa educativa y socializadora, por lo que es posible partir de una concepción ecológica de la lengua entendida en el marco de las relaciones que se establecen entre los individuos y su entorno.

Admitida, pues, la impronta mutua que dejan lengua y cultura sobre la construcción ideológica de los individuos de un determinado marco cultural, podemos avanzar y observar cómo esa impronta se evidencia en las distintas manifestaciones discursivas orales y escritas que conforman parte del patrimonio cultural de una sociedad.

Desde luego, en el estudio del sexismo lingüístico se ha de tener presente la perspectiva que proporcionan los estudios críticos del discurso. La razón por la que emplearemos esta perspectiva de análisis es, fundamentalmente, porque, como señala van Dijk (1999: 23) esta orientación toma partido, es decir, espera contribuir a la resistencia contra la desigualdad social y se puede entender como una reacción contra los paradigmas formales dominantes. En el caso que nos ocupa será determinante adoptar esta perspectiva de análisis tanto para valorar producciones textuales como el informe de Bosque, como para entender y analizar correctamente los aspectos gramaticales y léxicos del español que permitirán sostener la idea del rechazo a una serie de actuaciones o intervenciones (Moreno Cabrera 2012) con el fin de eliminar la desigualdad social manifestada a través de la lengua. En la última sección del apartado 6 vincularemos esta perspectiva al análisis textual del último diccionario usual académico con el fin de poner de manifiesto las estrategias discursivas que se observan en esta edición y en sus enmiendas.

Además de la estructura polarizada dominante que describe van Dijk (2010: 26) para el caso del sexismo lingüístico (Nosotros-hombres frente a Ellas-mujeres); en el estudio del triángulo que conforman mujer, lengua e ideología, resulta de gran ayuda acudir a algunos trabajos clásicos del feminismo como el de Buxó Rey (1978). En el referido estudio se introducen interesantes conceptos, todavía vigentes en lo esencial, como el de la asimetría sexo-social que se materializa en el comportamiento sexolingüístico. Para esta autora, el sistema lingüístico se sustenta en sistemas simbólicos 
arraigados en el inconsciente profundo de la cultura que buscan mantener la forma tradicional de la estructuración social que gobierna las relaciones entre ambos sexos. Buxó Rey (1978: 97) se detiene también en señalar tres aspectos gramaticales y léxicos presentes en muchas lenguas que contribuyen a sostener esta asimetría entre hombres y mujeres. Por su vinculación con los aspectos que serán tratados en este estudio, y con el fin de demostrar la reivindicación que desde hace más de tres décadas se lleva realizando con respecto al sexismo lingüístico, conviene recordar esos tres aspectos. El primero se refiere al uso del masculino como genérico o como forma no marcada, que indica, según Buxó, la superioridad del hombre. El segundo remite al uso androcéntrico de la gramática prescriptiva, por ejemplo en el sistema pronominal. Por último, el tercer aspecto, que es de índole léxico-semántica, se refiere a los repertorios lexicográficos, en los que ella entiende (1978: 98) que se incluyen "distinciones semánticas adicionales que son vivo reflejo de connotaciones sociales diferenciadas para ambos sexos”. Desde luego, estos puntos no acusan en absoluto obsolescencia si nos detenemos a analizar las nuevas obras gramaticales y lexicográficas realizadas al amparo de la RAE, como demostraremos en este artículo.

Por otra parte, en cuanto a la relación que se establece entre discurso y dominación, como defiende Pardo Abril (2001: 173) existen varias dimensiones discursivas desde las que los hablantes de una lengua construyen formas de dominación. La primera remite a la dominación que se ejerce a través de las representaciones sociales que rodean al discurso, cuyo poder se ejecuta en el uso de determinadas estructuras lingüísticas que consiguen manipular los modelos mentales de los interlocutores. La segunda se relaciona con la apropiación de contextos específicos de interacción, es decir, el dominio se ejerce a través de la limitación de los derechos comunicativos de los demás. Nos interesa en nuestro análisis la primera dimensión descrita, pues el sexismo lingüístico en la lengua española no se manifiesta tanto en la restricción de soportes comunicativos para la mujer, como en el uso de recursos lingüísticos, fundamentalmente gramaticales y léxicos, que veremos a lo largo de este estudio.

\section{LENGUA Y CONOCIMIENTO DEL MUNDO}

En este apartado nos ocuparemos de la relación entre conocimiento lingüístico y conocimiento del mundo, lo cual remite en último término a la relación entre lengua y cultura. Este punto de partida nos permitirá seguir una línea argumentativa para rastrear las posibles motivaciones semánticas que actúen, bien en la conformación de la categoría gramatical de género en las lenguas o bien en su funcionamiento. Esto no implica que todos los nombres de una lengua como el español estén motivados en cuanto al género, sino admitir que algunas nociones que sustentan la distinción genérica en ciertas partes del vocabulario (en particular, nombres que sirven para diferenciar entre mujeres y hombres) pueden contribuir a mantener viva esa base semántica y alimentan el uso creativo a través de procesos de reasignación de género. Por ejemplo, el originariamente femenino la centinela ha formado un masculino el centinela por su uso habitual históricamente para designar varones. ${ }^{1}$ En otros casos,

\footnotetext{
En el diccionario de Autoridades (1729) figura como sustantivo femenino. En el diccionario usual de la RAE de 1780, si bien aparece caracterizado como femenino, después de la definición se lee: "Úsase también como masculino. En lo antiguo se usó como femenino" (RAE 2001b).
} 
la formación de masculinos o femeninos analógicos va más allá, pues altera la desinencia original, por ejemplo en el modisto (ejemplos de Lledó 2012) o, en sentido inverso (esto es, del masculino al femenino) en la jueza o la presidenta.

Todavía hoy en día persiste la opinión según la cual la asignación de los nombres a cada uno de los miembros de la categoría de género tiene carácter arbitrario (por ejemplo en Roca 2005: 26). Si bien es cierto que la mayoría de los nombres no muestran un contraste morfológico (del tipo tío - tía) y que los contrastes fónicos del tipo /a/ - /o/ en el margen derecho de las palabras no siempre se correlacionan con referentes que en el mundo real puedan ser adscribibles a un género u otro desde el punto de vista biológico o el social (el templo, la montaña, la mano), ha de tenerse en cuenta que en español (como en otras lenguas europeas occidentales) el género es un sistema de clasificación que no se agota ni mucho menos en su función de distinguir entre hombres y mujeres (o a animales machos y hembras), sino que todos los nombres, con independencia de que hagan referencia a entidades animadas o inanimadas, se adscriben a un género o a otro, sin que exista uno "residual", esto es, que sirva para agrupar a los conceptos que no son "ni lo uno ni lo otro". El carácter omnipresente de la categoría no excluye, sin embargo, que esté vivo en nuestra conciencia lingüística el recurso del género para señalar, cuando lo creemos preciso, si nos estamos refiriendo a mujeres o a hombres.

Entre quienes opinan que el género suele tener una motivación está Dixon: “es un hecho empírico que las clases nominales parecen tener siempre algún grado de correspondencia semántica"2 (1982: 173). Esto puede ir más allá, motivando asociaciones de género en inanimados que, en ocasiones, pueden tener una base cultural, reflejando actitudes y valores culturales (cfr. Jakobson 1971: 265).

Langacker, con su casi equiparación entre estructura semántica y estructura conceptual (Langacker 1987: 154), concibe nuestro conocimiento lingüístico como esencialmente enciclopédico. Su visión cognitivista supone aceptar que las palabras y las expresiones codifican algo más que una representación abstracta y esquemática de los conceptos denotados. Añadiremos que en esta concepción la gramática es el resultado de un proceso de sedimentación (entrentchment), rutinización o habituación psicológica (Langacker 2000: 93) a través del cual se ritualizan ciertas asociaciones de forma y contenido.

Esta visión de la gramática es coherente con un modelo basado en el uso, en el cual la repetición de determinadas formas (más frecuentes) proporciona la base explicativa para una gramática emergente (Bybee y Hopper 2001), también concebida como el resultado de procesos de sedimentación.

Por otra parte, Lakoff (1987) proporciona una explicación semántica para la asignación de los conceptos a cada una de las cuatro clases semánticas del chilbal (lengua australiana), siguiendo la descripción de Dixon (1982). Para ello utiliza un modelo de categorización radial, con una subcategoría central ('central subcategory') y extensiones no centrales ('noncentral extensions') (1987: 91).

El interés de la propuesta de Lakoff radica en que proporciona explicaciones de base semántica para una organización en clases gramaticales que es en esencia de género. Aunque no es extrapolable a otras lenguas, constituye en sí misma un ejemplo de cómo las asociaciones de significado enraizadas en la cultura actúan para configurar la categoría.

"[I]t is an empirical fact that noun classes seem always to have some degree of semantic correspondence" (Dixon 1982: 173). El autor considera al género como una realización particular de las clases nominales. 
Ambadiang propone una clasificación de los nombres animados del español basada en el sexo de sus referentes (1999: 4848-4851), y recoge algunas clases léxicas de nombres inanimados en las cuales se pueden apreciar algunos criterios semánticos. Para estos últimos, los factores semánticos son menos sistemáticos, y presentan numerosos conflictos e interferencias (Ambadiang 1999: 4851-4853)

\section{LA ASIGNACIÓN DEL GÉNERO EN LAS LENGUAS Y LOS CONFLICTOS DE RESOLUCIÓN SINTÁCTICA}

\subsection{Asignación de género}

Siendo el género una categoría que clasifica los nombres en las lenguas, cabe preguntarse con qué criterio se reparten estos entre las clases por ella establecidas, en particular en qué medida pesan interlingüísticamente los factores semánticos en la clasificación de los nombres en diferentes géneros. Corbett (1991) llama a esto asignación de género ('gender assignment'). Los criterios en juego son semánticos o formales. ${ }^{3}$

Según el autor, todos los sistemas de género son semánticos en el sentido de que siempre existe un núcleo a partir del cual reconocer una asignación semántica (Corbett 1991: 8). Pero en las lenguas con asignación semántica propiamente dicha es posible saber si un nombre pertenece a uno u otro género solo con conocer su significado.

El sexo biológico es solo uno de los criterios semánticos en que se basa la distinción del género en las lenguas. Corbett (1991: 30-31) cita, entre otros: animado / inanimado, humano / no humano, macho / hembra, grande / pequeño, comestible o insecto. No obstante, son muchos los sistemas de género ligados al sexo biológico. Es frecuente que cuando hay una base semántica este sea uno de los criterios.

El autor describe una escala en uno de cuyos extremos están las lenguas con un alto grado de correspondencia entre la categoría gramatical y la designación. Así, en las lenguas drávidas (por ejemplo, el tamil), para la cual se puede afirmar casi con total seguridad que los nombres que denotan humanos machos son masculinos y los nombres masculinos denotan humanos machos. En español, en cambio (como en francés o ruso, que son las lenguas con las que ejemplifica el autor), los nombres que denotan hombres o animales machos son típicamente masculinos (Ambadiang 1999: 4848), pero hay un gran número de nombres masculinos que tienen otras designaciones, en particular de seres u objetos asexuados. Además, algunos nombres que denotan seres sexuados están en el "género equivocado", si bien en estos casos la concordancia puede no seguir la pauta formal (Corbett 2011b). Por tanto, el español está entre las lenguas que asignan el género con criterios de sexo, pero esto solo explica una parte nuclear (un 'semantic core', Corbett 1991: 57), pues un número importante de los nombres se asignan con criterios formales, esto es, el hecho de que sean femeninos o masculinos no se deduce de su significado, sino de aspectos formales, como es el hecho de que tengan una u otra desinencia.

Las controversias sobre el sexismo en el lenguaje tienen que ver con esa parte del vocabulario que constituye la parte nuclear que permite justificar la decisión de Corbett de que el género en español se sustenta semánticamente en la distinción de sexos. El 'semantic core' es el conjunto de nombres para el cual es relevante -o puede

\footnotetext{
Corbett (2011a) proporciona recuentos sobre lenguas con y sin género gramatical. Para las que sí tienen distinción género, ofrece datos de lenguas con asignación solo semántica, o bien semántica y formal. En Corbett (2011b) observa el número de los sistemas de género basados en el sexo y lo compara con los no basados en el sexo.
} 
serlo- el saber si hablamos de mujeres o de hombres. Se podrá decir que esta no es una cuestión pertinente desde el punto de vista lingüístico, pero sí lo es en la medida en que la distinción se codifica en la lengua.

\subsection{Resolución de género}

Para hablar de género en tanto que categoría gramatical, no solo se requiere que los nombres se repartan en distintas clases, además esta división producirá algún efecto reconocible como concordancia. Así, el género o clase a que pertenece una forma controlada por concordancia (por ejemplo, en un adjetivo) dependerá de algún aspecto (semántico o formal) de la forma controladora. Ahora bien, en algunos contextos sintácticos esto puede producir un conflicto, por ejemplo, si se coordinan nombres que pertenecen a clases (géneros) diferentes. Los criterios que resuelven para cada lengua cuál es el género que toma la forma controlada se llaman de resolución de género (o reglas de resolución de género, 'gender resolution rules').

La resolución del género puede seguir criterios semánticos, como en la lenguas dravídicas y en las bantúes, o sintácticos (francés, esloveno, español, letón, hindi, penyabí, hebreo moderno; ver Corbett 1991:280). También existe una resolución mixta (polaco, ibid: 284ss). Como en el caso de la asignación de género, si los criterios semánticos imperan, el resultado será que se optará por el género que se imponga a través de la denotación. En las lenguas en que funcionan criterios sintácticos, en cambio, el género de la forma controlada dependerá de las formas controladoras, con independencia de su referencia.

Corbett defiende que, aunque el mecanismo de resolución del género es sintáctico, la motivación es semántica y funcional (284 y sección 9.6). Para determinar cuál ha de ser el término que aparezca en los casos de resolución sintáctica, pone de relieve el fenómeno de la dominancia, el uso de un género en lugar de los dos, como es el caso del español los padres para referirse conjuntamente al padre y la madre, o el francés les américains (masculino) también para denotar hombres y mujeres de esa nacionalidad (Greenberg 1966: 30-31, Corbett 1991: 291). Esto supone dar mayor peso a los factores semánticos, en particular a la posibilidad de que uno de los términos de la categoría pueda usarse para una referencia conjunta. En el siguiente apartado analizaremos esta capacidad del masculino de hacer referencia conjuntamente a seres masculinos y femeninos.

\section{El SEXISMO LINGÜÍSTICO EN LA LENGUA ESPAÑOLA: ASPECTOS GRAMATICALES. El MASCULINO GENÉRICO COMO TÉRMINO NO MARCADO EN ESPAÑOL}

En español el género clasifica los nombres en dos grandes grupos: masculinos y femeninos. Además, tiene efectos en la concordancia, particularmente en los adjetivos y en algunos determinantes. Se suele decir que el femenino es el género marcado (Arias Barredo 1990, Aliaga García y Lázaro Mora 2003). ${ }^{4}$ La defensa del masculino

No obstante, Aliaga García y Lázaro Mora defienden que esto no es así en todos los casos, pues en ocasiones es el género femenino el que opera como no marcado. El ejemplo que ponen es "Odio las palomas" (Aliaga García y Lázaro Mora 2003: 10). Esto se debe a que definen el fenómeno de la marcación con el criterio de que opera únicamente "entre parejas de nombres que denotan seres sexuados de la misma clase" (ibid: 5). Parecen preferir esta concepción a una de base estrictamente gramatical, a pesar de que son conscientes de que esta es menos restrictiva y les permitiría abarcar los casos de uso del masculino por resolución de conflictos de género, como en "Juan y mi prima María son extremadamente simpáticos/*simpáticas" (ibid: 8). 
genérico como término no marcado suele justificar la de su uso -en contra de los desdoblamientos- con un significado referencial que engloba personas femeninas y masculinas. La encontramos también en RAE (2009: §2.2a).

La RAE ejemplifica con un caso de heteronimia, es decir, de distinción de género con base léxica (el sustantivo hombre) y lo hace extensivo también a los animales (ibid.).

La idea de que el masculino actúa como género no marcado descansa en las siguientes observaciones:

Puede tener una interpretación que equivalga en su denotación a la de nombres masculinos y femeninos conjuntamente. El femenino, en cambio, solo admite la interpretación más restrictiva. Arias Barredo (1990: 114; citando a su vez a Echaide 1969) ofrece los siguientes ejemplos:

(1) a. El segundo hijo que ha dado a Avito Marina ha sido hija

b. El madrileño 3.000.000 [...] ha sido madrileña

Y argumenta que no es posible la inversión de los términos hijo e hija, madrileño y madrileña, lo cual prueba que el femenino no admite la interpretación genérica:

(2) a. *La segunda hija que ha dado a Avito Marina ha sido hijo

b. *La madrileña 3.000.000 [...] ha sido madrileño

El género no marcado puede no codificarse con una forma ostensible, lo que sí ocurrirá en cambio en el caso del género marcado. Este criterio solo valdrá para aquellos nombres en que para formar el femenino se añade una desinencia sobre el masculino, que se interpretará como la raíz o forma básica: león-leona, tigre-tigresa, poeta - poetisa, abad - abadesa. Cuando el masculino y el femenino de los nombres puedan asociarse a una desinencia diferente (una para cada uno), no será factible esta interpretación, salvo que tengan diferente "peso" fonológico a favor del femenino.

Aparece en más contextos que el femenino, de tal manera que se puede interpretar que es la forma preferida en casos de "neutralización" (en el sentido de Trubetzkoy 1939; vid. Haspelmath 2006), es decir, en los casos en que se suspende la oposición de género y solo uno de los miembros de la categoría es posible. Caben en esta interpretación los siguientes ejemplos, aducidos por Bosque (2012):

\section{(3) a. Juan y María viven juntos \\ b. Juan y María se ayudan el uno al otro en lo que pueden \\ c. Ayer estuvimos comiendo en casa de mis padres}

En (3a y 3b) la elección del masculino viene determinada por razones sintácticas y en (3c) es un fenómeno léxico (en español el término para la pareja de madre y padre es igual al plural del masculino específico padre). (3c) es un caso de "dominancia" (un género vale por los dos) y ( 3 a y 3 b) son de "concordancia a potiori", definida por Greenberg en los siguientes términos: "palabras que seleccionan dos o más categorías como el género comparten un modificador y el modificador está en la categoría no marcada, por ejemplo en español el hijo y la hija son buenos”' (Greenberg 1966: 31).

"words from two or more selective categories such as gender have a common modifier and the modifier is in the unmarked category, e.g. Spanish el hijo y la hija son buenos" (Greenberg 1966: 31). 
Como consecuencia de lo anterior, el masculino es más frecuente que el femenino (con el doble de apariciones, según Arias Barredo, quien cita de nuevo a Echaide como fuente).

Haspelmath (2006) revisa los diferentes sentidos en que se emplean los términos marcado y no marcado en lingüística y concluye que la noción de marca ('markedness') es prescindible y que contribuye poco a comprender los fenómenos a que hace referencia. Argumenta que estos se pueden explicar a través de algún otro concepto. En este trabajo solo nos ocuparemos de una selección de las acepciones de la marca que son aplicables a la distinción de género.

En concreto, para la denotación conjunta a la que hacíamos alusión en (a) habla del sentido de marca semántica ('semantic markedness'; Haspelmath 2006: $\S \S 2.1 .2$ y 5). Aduce que los conceptos semánticos de hiponimia y polisemia pueden sustituir a este empleo de marca y marcado. El término genérico (hijo, madrileño) se comporta como un hiperónimo con respecto al específico (hija, madrileña). ${ }^{6}$ Los ejemplos que proporciona el autor no son de género gramatical, sino de diferencias léxicas no siempre correlativas con el género, pero nos pueden servir para entender el mecanismo de la ampliación de significado que el masculino representa con respecto al femenino si les añadimos algún ejemplo de contraste entre masculino y femenino. Estos ejemplos, bajo la forma de pares de términos definibles como genérico (término 1) frente a específico (término 2), son sometidos a varios criterios, que permiten dar una serie de valores a cada uno de ellos. Adaptamos parcialmente la tabla de Haspelmath (2006: $\S 5)$, tomando solo algunos de sus ejemplos, en tanto que añadimos algunos casos más de oposición de género en español y respetamos los mismos criterios que él emplea, así como también la forma de anotar los valores. El resultado es la tabla 1. Para los términos que respetamos del original (vehicle - bus, lion - lioness, cow - bull, house church, Arzt - Ärztin (alemán 'doctor - doctora'), man - woman, king - queen, padre - madre), mantenemos los mismos valores que les da el autor.

Estamos de acuerdo con Haspelmath en que la tabla muestra una diversidad de comportamientos difícilmente explicable a través del concepto de marca en la acepción de diferencias de denotación entre el término 1 y el 2, pues son las propiedades idiosincráticas de las palabras las que a fin de cuentas explican la heterogeneidad de los valores. Ahora bien, al tratar de extender el análisis a la diferencia de género en español los resultados no son muy diferentes, en el sentido de que no parece que se pueda deducir de la oposición masculino - femenino las diferencias de comportamiento de los pares de términos. Si bien alumno - alumna parece dar los mismos resultados que hijo - hija, no ocurre lo mismo con abad abadesa y esposo - esposa, de lo cual se deriva que también en estos casos existen peculiaridades léxicas que deben tenerse en cuenta a la hora de valorar las diferencias en cuanto a la forma de manifestarse el uso de la forma genérica, el masculino. No tiene sentido decir Este abad es una abadesa, ni tampoco que una abadesa sea un tipo particular de abad. Incluso la prueba del plural heterogéneo es más que dudosa, pues no parece sencillo encontrar un contexto en que abades englobe conjuntamente a los términos específicos masculino y femenino. El plural heterogéneo sí se usa

Roca (2006) interpreta la relación semántica entre masculino y femenino como hiperonimia en ejemplos como esposo, religioso o ciudadano. Para ese autor, estas palabras no tienen "determinación de sexo" (2006: 409). Es decir, en ausencia de la "marca opcional" de femenino, habrán de referirse conjuntamente a hombres y mujeres. Desde esta concepción es sorprendente que hijo en Ya ha llegado el hijo de María se interprete como "solo varón", aunque sea esta la interpretación habitual (Roca 2006: 414). 
en esposos (no muy diferente, a nuestro juicio, de padres). Como en este último, también en esposos se espera una interpretación por defecto que denote a una pareja, lo cual dificulta otras manifestaciones del uso genérico.

Tabla 1. Rasgos semánticos de la hiponimia. Análisis basado en Haspelmath (2006)

\begin{tabular}{|c|c|c|c|c|c|c|c|}
\hline $\begin{array}{l}\text { Término } 1 \\
\text { (=genérico) }\end{array}$ & $\begin{array}{c}\text { Término } 2 \\
\text { (=específico) }\end{array}$ & $\begin{array}{c}\text { Se excluye la } \\
\text { predicación con- } \\
\text { trastiva ('Esto es } \\
\text { un } 1, \text { no un 2') }\end{array}$ & $\begin{array}{c}\text { Uso específico } \\
\text { de } 1 \text { por } 2 \text { ('Vi } \\
\text { un 1' significa } \\
\text { 'Vi un 2') }\end{array}$ & $\begin{array}{l}\text { 'Este 1 } \\
\text { es un 2' }\end{array}$ & $\begin{array}{l}\text { Hiponimia ('2 } \\
\text { es un tipo parti- } \\
\text { cular de 1') }\end{array}$ & $\begin{array}{c}\text { Uso no especí- } \\
\text { fico de } 1 \text { por } 2 \\
\text { ('Busco un } 1 \text { ' } \\
\text { incluye a } 2 \text { ) }\end{array}$ & $\begin{array}{c}\text { Plurales } \\
\text { hetero-géneos } \\
\text { ('1s' vale por } \\
\text { '1s y 2s') }\end{array}$ \\
\hline Vehicle & bus & Sí & Sí & Sí & Sí & Sí & Sí \\
\hline Lion & lioness & ¿? & Sí & Sí & Sí & Sí & Sí \\
\hline Cow & Bull & - & $\dot{¿} ?$ & $\dot{¿} ? ?$ & ¿? & $\dot{¿} ?$ & Sí \\
\hline House & church & - & ¿? & ¿?? & $\dot{¿} ? ?$ & $\dot{i}^{? *}$ & ¿? \\
\hline Arzt & Ärztin & - & - & - & $\dot{c} ?$ & Sí & Sí \\
\hline Man & woman & - & - & - & - & - & (Sí) \\
\hline King & queen & - & - & - & - & - & - \\
\hline Padre & madre & - & - & - & - & ${ }_{-}^{1}$ & Sí \\
\hline Hijo & hija & - & - & Sí & Sí & Sí & Sí \\
\hline Alumno & alumna & - & - & Sí & Sí & Sí & Sí \\
\hline Abad & abadesa & - & - & - & $\dot{¿} ?$ & $\dot{¿} ?$ & - \\
\hline Esposo & esposa & - & - & - & - & - & Sí \\
\hline
\end{tabular}

${ }^{1}$ En este criterio (uso no específico de 1 por 2) discrepamos del análisis de Haspelmath, pues creemos que es posible, en un contexto propicio (en una reunión del colegio, por ejemplo), decir Busco un padre utilizando este término de manera genérica, esto es, con la expectativa de que encontrar una madre satisfaga las condiciones pedidas.

Haspelmath (2006: §5) explica los fenómenos de “marcación semántica” a través de extensiones de cierta interpretación pragmática. Así, en la lengua maidu de California, majdy significa "ser humano" (genérico), pero también "persona maidu" (específico), es decir, es el término 1 con respecto a formas "marcadas" como kombom majdy "persona yana", o wolem majdy "persona blanca", que actuarían como un término 2 de nuestra tabla, dotado únicamente de significado específico (ejemplo de Greenberg 1966: 26). El uso de majdy como "persona maidu" es una interpretación "por excelencia."7 Puesto que lo habitual en esa cultura es que se hable de personas maidu, cuando no sea ese el caso se dirá de otra manera, una que elimine la expectativa por defecto.

Similares implicaturas pragmáticas (o, más bien, las expectativas de uso que su consolidación propicia) ayudan a comprender algunos casos que parecen revelar una tendencia a interpretar preferentemente como denotaciones específicamente masculinas algunos usos pretendidamente genéricos del masculino de la lengua española. Los ejemplos (4a-c) son de García Meseguer (1994) y (4d) está adaptado de la guía de la UGT $(2008)^{8}$ :

Greenberg (1966: 66) explica en términos de frecuencia la referencia masculina "por excelencia" de la palabra inglesa author, así como también la interpretación por defecto de nurse como de referencia femenina.

8 En el original: "Los directivos acudirán a la cena con sus mujeres". Reproducido así en Bosque (2012). En ambos documentos se considera este uso como sexista. 
(4) a. El hombre / el coyote es un mamífero y por ello amamanta a sus crías

b. El hombre es el único animal que menstrúa

c. Pedro tiene un lío amoroso con un familiar mío

d. Los directivos acudirán a la cena con sus esposos

La extrañeza que suscitan ejemplos como los de (4), ${ }^{9}$ unidos a las matizaciones sobre la "hiperonimia" del masculino genérico que sugiere la exposición a los criterios de Haspelmath de algunos casos como los de abad - abadesa y esposo - esposa, invitan a una cierta prudencia ante las afirmaciones categóricas sobre el uso del masculino en el caso de nombres animados "para designar la clase que corresponde a todos los individuos de la especie” (RAE 2009: §2.2a) y, sobre todo, a la hora de desacreditar el uso de los desdoblamientos del tipo "los directivos y las directivas", así como de expresiones de referencia "neutra", como cónyuges o parejas (preferibles a esposos, en $7 \mathrm{~d}$ ).

El criterio (b) (forma ostensible del término marcado) se corresponde con el sentido que llama Haspelmath (2006: $\S \S 2.3 .1$ y 4.3) de marca formal ('formal markedness'). Hemos señalado ya que es un criterio bastante limitado en su aplicación al género gramatical. Haspelmath prefiere explicar esta acepción del concepto de marca en términos de frecuencia de uso y de longitud fonética. Esto es, el hecho de que una forma sea más frecuente que la otra tiene como consecuencia que su realización fónica resulte ser más breve. Una consulta en el banco de datos CREA de la Real Academia Española confirma esta correlación entre término más explícito en su marca y menor frecuencia. No obstante, las diferencias de frecuencia entre el término masculino y el femenino no muestran correlaciones estables, lo cual es esperable dado que se trata de lexemas diferentes y que no hemos hecho ninguna restricción sobre los contextos de uso. La primera cifra corresponde a la frecuencia absoluta y la segunda (entre paréntesis) la frecuencia normalizada:

(5) a. Abad: $1.041(6,82)$ - abadesa: $213(1,39)$

b. León $10.541(69,09)$ - leona: $512(3,35)$

c. Poeta: $9.572(62,74)$ - poetisa: $299(1,95)$

d. Tigre: $1.488(9,75)$ - tigresa: $58(0,38)$

La mayor frecuencia de aparición del llamado término no marcado coincide con el criterio (d) de los que exponíamos más arriba y es la interpretación que Haspelmath prefiere sobre todas las demás posibles explicaciones a este tipo de fenómenos. ${ }^{10} \mathrm{Se}$ refiere a ella como rareza en los textos ('markedness as rarity in texts'). Podemos extender la consulta del CREA a otros ejemplos en que no haya diferencia en la longitud de los dos géneros. Insistimos en las limitaciones de una búsqueda "plana",

9 Que también son explicables como efectos de prototipo (Lakoff 1987), donde el prototipo sería una representación cognitiva idealizada de hombre en tanto que varón, coyote como macho de la especie, etc.

10 Greenberg (1966: 66-67) defiende ya las ventajas de una interpretación del concepto de marca en términos de frecuencia, aunque no pretende que sea sustituido por este. 
es decir, en ausencia de datos referidos al contexto sintáctico y semántico. ${ }^{11}$ Como más arriba, se indica primero la frecuencia absoluta, y luego, entre paréntesis, la frecuencia normalizada:

(6) a. Padre: $45.236(296,51)$ - madre: $43.697(286,42)$

b. Hijo: $30.298(198,59)$ - hija: $15.958(104,60)$

c. Alumno: $2.969(19,46)$ - alumna: $460(3,01)$

d. Abogado: $8.983(58,88)$ - abogada: $740(4,85)$

Sin duda, el hecho de que el masculino sea el término más frecuente se relaciona con que sea también el seleccionado en los casos de resolución sintáctica. ${ }^{12}$

Nuestra característica (c) del término no marcado como el que aparece en contextos de neutralización coincide con el sentido al que se refiere Haspelmath (2006: § 2.3.4) de la marca como distribución restringida ('markedness as restricted distribution'). Aunque nació de la fonología funcional europea, es en el terreno de la sintaxis donde el autor reconoce cierta utilidad, en la medida en que no parece poder reducirse a otra dimensión diferente. ${ }^{13}$ Más arriba describíamos también como sintácticos los casos en que esta acepción es útil para dar cuenta de ciertos fenómenos que ocurren en el género del español: aquellos en que el término masculino -ya sea en un nombre o en un adjetivo- aparece exigido por fenómenos de concordancia y además existe algún "conflicto" de los dos tipos a los que nos hemos referido como "dominancia" y como “concordancia a potiori". Ver ejemplos de (3) supra.

Que el masculino es el término preferido por la gramática del español en casos de resolución sintáctica está también reforzado por la selección que imponen aquellas formas gramaticales que no tienen género. Sirvan de ejemplo las expresiones de (7), con cláusula completiva (7a) y (7b) y de infinitivo (7c). El primero reproduce esquemáticamente el principio de este mismo párrafo:

(7) a. Que el masculino es el término preferido (...) está reforzado (...)

b. Es curioso que hayan tenido en cuenta incluso eso. (SEV: 134, 04)

c. fue necesario reparar con fondos públicos la puerta principal (CRO: 054, 03) ${ }^{14}$

Ni en los ejemplos de (3) ni en los de (7) es posible el femenino, con la única excepción parcial de (3b), que permite Juan y María se ayudan el uno a la otra en lo que pueden, pero no transmite las mismas implicaturas pragmáticas. Por tanto, la

11 Debe tenerse en cuenta, además, que la aparición de una determinada forma en el corpus vendrá determinada por multiplicidad de factores, entre los que es determinante la selección de textos que han dado lugar al mismo. Hemos evitado el par madrileño - madrileña porque es un adjetivo y su referencia no es necesariamente humana. En el caso de esposo - esposa, cuyas frecuencias sería muy interesante conocer, tenemos el problema de que el femenino es homónimo con la tercera persona del presente del verbo esposar.

12 Corbett lo achaca al fenómeno de la dominancia. Sin duda, estamos ante una correlación de factores que privilegian una determinada selección. Este autor es poco proclive también a considerar la marca como factor determinante de los fenómenos de resolución de género por su escasa relevancia interlingüística (Corbett 1991: 292).

13 En el caso de la distribución defectiva de los fonemas, se puede acudir a una explicación sustancial: la mayor dificultad de pronunciación del término que no aparece en todos los contextos (Haspelmath 2006: § 2.3.4).

14 Ejemplos procedentes de ADESSE. 
resolución sintáctica del género en español se puede describir en término de que el masculino está distribucionalmente no restringido y actúa "en los demás casos".

\section{El SEXismo lingüístico en la lengua española: asPectos léXicos (el DRAE)}

\subsection{Los pares morfológicos}

La incorporación de la mujer y del hombre a ciertos ámbitos profesionales, tanto civiles como militares, en el ámbito panhispánico ha motivado la creación de nuevos pares morfológicos para nombres de profesiones y cargos atribuidos tradicionalmente a uno u otro sexo. La particularidad que el español presenta en relación con estos pares morfológicos es la falta de sistematicidad y de revisión con la que muchos de ellos se recogen en las obras lexicográficas publicadas por el ente rector en materia normativa, es decir, en los diccionarios de la RAE. También es característica la discordancia entre la norma y el uso real, como demuestran estos ejemplos extraídos de titulares y textos de la prensa digital e impresa en los que sí se recogen algunas formas a las que el DRAE todavía no da cabida:

(8) Jequesa

a. El turbante hipnótico de la jequesa de Catar (El País, 26/04/2011).

b. La jequesa de Qatar deslumbra con un diseño de J. Mendel (ABC, $10 / 04 / 2012)$

c. La jequesa de Qatar deslumbra en España (El Mundo, 25/04/2011).

(9) Cancillera

a. La mala cara de la cancillera (El Periódico, 15/05/2012).

b. Los socialdemócratas derrotan a la cancillera Merkel en Renania del Norte (PressDigital, 13/05/2012).

c. En manos de la cancillera Merkel (El Periódico de Aragón, 18/06/2011).

(10) Estibadora

a. La vida en el muelle de las estibadoras. Son las primeras mujeres que hacen este trabajo y están en Málaga (Diario Sur, 03/06/2007).

b. Arribó al puerto gaditano la primera mujer estibadora (Diario de Cádiz, 06/05/2012).

c. Una estibadora conducía maquinaria pesada en Valencia con el carné retirado (Las Provincias, 08/01/2011).

(11) Soldadora

a. Sólo hace tres años salió del Centro de Nuevas Tecnologías de Gijón (Asturias) la primera mujer soldadora (El País, 08/12/2002).

b. El naval de Vigo incorpora a la primera cuadrilla de mujeres soldadoras (La Voz de Galicia, 04/03/2007).

c. Una brigada de mujeres soldadoras entrará por primera vez en AZSA

(La Voz de Asturias, 12/02/2010).

Desde luego, se trata de una muestra mínima, pero no deja de resultar sorprendente la celeridad con la que se han incorporado los pares masculinos de algunas profesiones 
tradicionalmente ocupadas por mujeres como matrón, modisto o azafato y la tardanza con la que se van incorporando los pares femeninos como los que proponemos en los ejemplos.

Como advierte Forgas Berdet (2011: 442), la Academia realiza una declaración de intenciones en las "Advertencias" del DRAE 2001 que finalmente no se cumplen en la práctica. Entre esas intenciones se encontraba la de llevar a cabo "un importante esfuerzo en cuanto al registro en masculino y femenino de determinados nombres referidos a profesiones, cargos y actividades con vigencia en la actualidad" (DRAE 2001: XXIX). Un análisis superficial de esta obra ya revela una realidad que difiere notablemente de estas intenciones, que en parte se está subsanando con las enmiendas propuestas para la vigésima tercera edición del DRAE (por ejemplo, alguacil, la).

Por otra parte, uno de los aspectos más interesantes del repertorio académico es la presencia de la obsoleta acepción "mujer de X (varón)" en multitud de lemas específicos para un género y en otros lemas con moción —entradas en las que se distingue flexión de género gramatical en función del sexo del referente- que se refieren normalmente a profesiones y cargos tradicionalmente ocupados por varones. Por supuesto, esto no sucede a la inversa, es decir, en ningún caso encontraremos "marido de Y (mujer)" cuando se defina la forma masculina de una profesión o cargo que remita a actividades profesionales desempeñadas tradicionalmente por mujeres (por ejemplo, matrón, na). Este hecho se refleja claramente en el caso de los títulos nobiliarios: sí encontraremos que la condesa es la mujer del conde, pero en ningún caso se registrará como acepción la de conde como "marido de la condesa". Asimismo, también se observará falta de sistematicidad en el proceder académico a la luz de la ausencia de este tipo de acepción "mujer de" en cargos como, por ejemplo, senadora y de su presencia en otros como gobernadora. Esta falta de rigor en las revisiones será una tendencia habitual en los trabajos lexicográficos académicos, no solo en el ámbito que nos ocupa sino también en cuanto a la revisión ideológica general. De hecho, Forgas Berdet (2011: 442) señala que, efectivamente, en la vigésima segunda edición se reunieron "en un solo lema entradas de profesiones en masculino y femenino que aparecían inexplicablemente desdobladas” como notario, ria o comadrón, na (en concreto, 59 entradas pasaron a convertirse en 29 lemas de entrada única); pero que, sin embargo, las actuaciones no entraron en la totalidad de los artículos lexicográficos, como demuestran los problemas que estamos destacando hasta el momento.

A continuación se recogen algunos ejemplos que evidencian esta obsolescencia e inmovilismo en el proceder académico, un proceder que manifiesta sin lugar a dudas un desajuste con respecto a la realidad social actual pese a la presencia generalizada de marcas pragmáticas:

(12) DRAE 2001alcaldesa. 2. f. coloq. Mujer del alcalde. No presenta enmienda para la $23^{\mathrm{a}}$ edición del DRAE.

(13) DRAE 2001 catedrático, ca. 4. f. coloq. desus. Mujer del catedrático. Enmienda DRAE $23^{\mathrm{a}}$ ed. catedrático, ca. 4. f. coloq. desus. Mujer del catedrático.

(14) DRAE 2001 doctor, ra. 5. f. coloq. p. us. Mujer del doctor. Enmienda DRAE $23^{\mathrm{a}}$ ed. doctor, ra. 4. f. coloq. p. us. Mujer del doctor.

(15) DRAE 2001 fiscala. 2. f. coloq. desus. Mujer del fiscal. No presenta enmienda para la $23^{\mathrm{a}}$ edición del DRAE. 
(16) DRAE 2001 gobernador, ra. 4. f. p. us. Mujer del gobernador. No presenta enmienda para la $23^{\mathrm{a}}$ edición del DRAE.

(17) DRAE 2001 jefe, fa. 2. f. coloq. p. us. Mujer del jefe. No presenta enmienda para la $23^{\mathrm{a}}$ edición del DRAE.

(18) DRAE 2001 jueza. 2. f. coloq. p. us. Mujer del juez. No presenta enmienda para la $23^{\mathrm{a}}$ edición del $D R A E$.

(19) DRAE 2001 maestro, tra. 22. f. coloq. p. us. Mujer del maestro. Enmienda DRAE $23^{\text {a }}$ ed. maestro, tra. 14. f. coloq. p. us. Mujer del maestro.

(20) DRAE 2001 médico, ca. 3. f. coloq. desus. Mujer del médico. Enmienda DRAE 23 $3^{\mathrm{a}}$ ed. médico, ca. 3. f. coloq. desus. Mujer del médico.

(21) DRAE 2001 ministro, tra. 14. f. coloq. p. us. Mujer del ministro. Enmienda DRAE $23^{\mathrm{a}}$ ed. ministro, tra. 14. f. coloq. p. us. Mujer del ministro.

(22) DRAE 2001 presidenta. 4. f. coloq. Mujer del presidente. No presenta enmienda para la $23^{\mathrm{a}}$ edición del DRAE.

En su informe "Sexismo lingüístico y visibilidad de la mujer", Bosque (2012: 8) sostiene que "nadie considera controvertida la propuesta de extender la formación de pares morfológicos a los nombres de profesiones y cargos"; no obstante, insiste en la idea de que determinadas profesionales optarán por privilegiar la forma masculina sobre la femenina. De este modo, parece que la convivencia del sustantivo común en cuanto al género (pensemos en juez o presidente) junto con las formas específicas de masculino y femenino (reflejadas a través de lemas con moción) partiría precisamente del uso real y de preferencias geográficas o personales.

Esta convivencia a la que hacemos referencia implica una serie de irregularidades en el diccionario académico que no pueden explicarse de otro modo que por la falta de rigor en cuanto a la aplicación de distintas pautas para la incorporación de pares morfológicos relativos a profesiones y cargos. Además, no solo la macroestructura del diccionario manifiesta la presencia de este tratamiento heterogéneo, también en la microestructura es posible detectar la falta de sistematicidad en algunos comentarios de carácter morfológico que se mantienen arbitrariamente en algunas definiciones (cfr. abogado, da). Veamos algunos ejemplos de estos comentarios morfológicos que, en general, suelen eliminarse en las propuestas de redacción para la vigésima tercera edición del DRAE:

(23) DRAE 2001 abogado, da. 1. m. y f. Licenciado o doctor en derecho que ejerce profesionalmente la dirección y defensa de las partes en toda clase de procesos o el asesoramiento y consejo jurídico. MORF. U. t. la forma en $\mathrm{m}$. para designar el $\mathrm{f}$.

Enmienda DRAE $23^{\mathrm{a}}$ ed. abogado, da. 1. m. y f. Licenciado en derecho que ofrece profesionalmente asesoramiento jurídico y que ejerce la defensa de las partes en los procesos judiciales o en los procedimientos administrativos. MORF. U. t. la forma en $\mathrm{m}$. para designar el f. Rosa es abogado.

(24) DRAE 2001 aparejador, ra. 2. m. y f. Técnico titulado que interviene con funciones propias en la construcción de edificaciones. MORF. U. t. la forma en m. para designar el f. Rosario es aparejador.

Enmienda DRAE 23 $3^{\mathrm{a}}$ ed. aparejador, ra. 2. m. y f. Técnico titulado que interviene con funciones propias en la construcción de edificaciones. 
(25) DRAE 2001 arquitecto, ta. 1. m. y f. Persona que profesa o ejerce la arquitectura. MORF. U. t. la forma en $\mathrm{m}$. para designar el f. Laura es arquitecto.

Enmienda DRAE 23a ed. arquitecto, ta. 1. m. y f. Persona que profesa o ejerce la arquitectura.

(26) DRAE 2001 ingeniero, ra. 1. m. y f. Persona que profesa la ingeniería o alguna de sus ramas. MORF. U. t. la forma en m. para designar el f. Silvia es ingeniero.

Enmienda DRAE 23a ed. ingeniero, ra. 1. $\mathrm{m}$. y f. Persona que profesa la ingeniería 0 alguna de sus ramas.

(27) DRAE 2001 médico, ca. 2. m. y f. Persona legalmente autorizada para profesar y ejercer la medicina. MORF. U. t. la forma en $\mathrm{m}$. para designar el $\mathrm{f}$.

Enmienda DRAE 23 ${ }^{\mathrm{a}}$ ed. médico, ca. 2. $\mathrm{m}$. y f. Persona legalmente autorizada para profesar y ejercer la medicina.

(28) DRAE 2001 perito, ta. 2. m. y f. ingeniero técnico. MORF. U. t. la forma en m. para designar el f. Asunción es perito.

Enmienda DRAE 23a ed. perito, ta. 2. m. y f. ingeniero técnico.

Mención aparte merece la jerarquía militar que, pese a la integración plena de la mujer en el ejército, no refleja los usos reales en el diccionario académico. La mayoría de los sustantivos que designan rango militar son en español comunes en cuanto al género o unigéneros masculinos, pero a medida que ascendemos en la jerarquía encontraremos divergencias con respecto a unas reglas que parecían generales. Se registra nuevamente en lemas como sargenta, tenienta, coronela, generala, almiranta o mariscala la acepción "mujer de", que acusa el carácter inveterado que ya destacamos con anterioridad. Asimismo, en lemas como tenienta se recogerá la acepción propia de la mujer con grado de teniente frente al resto de rangos militares en los que esta acepción no se registra. Por otra parte, en algunos casos, como en capitán, na se creará el par morfológico (aunque con comentarios adicionales en los que se indica la mayor frecuencia de uso de la forma masculina para referirse a mujeres) frente a los restantes rangos en los que no se llevará a cabo tal procedimiento.

\subsection{El uso de 'hombre' en el DRAE 2001 para referirse a mujeres y hombres}

Bosque (2012: 15) defiende en su informe que el uso del término hombre con carácter genérico que abarca a hombres y a mujeres es habitual en distintas lenguas, lo cual por cierto no legitima la pervivencia de este uso en el español. Efectivamente, esto es así; de hecho, este fenómeno de fosilización lingüística recibe, como Bosque también señala, el nombre de gramaticalización. Este uso abarcador al que hacemos referencia se entiende, pues, como un proceso mediante el cual la palabra hombre pierde su contenido significativo originario y se convierte en un elemento gramatical y, por lo tanto, a priori, carente de implicaciones ideológicas.

Sin embargo, observamos que este planteamiento del académico no se corresponde con la realidad de las actuaciones lexicográficas que se vienen llevando a cabo en el $D R A E$ desde hace unos años; actuaciones que buscan erradicar en la medida de lo posible la presencia de un uso en el que multitud de personas no se ven reflejadas o representadas. Es el caso de alma, que hasta el DRAE de 2001 mantenía en su primera acepción la referencia a que constituía la esencia del hombre. Muchas fueron las definiciones que se modificaron desde la edición de 1992, pero la falta de revisión 
rigurosa del repertorio académico incide de nuevo en la supresión o mantenimiento arbitrarios de esta palabra en algunas definiciones, como demuestran estos ejemplos:

(29) DRAE 2001 cabeza. 1. f. Parte superior del cuerpo del hombre y superior o anterior de muchos animales, en la que están situados algunos órganos de los sentidos e importantes centros nerviosos. 3. f. En el hombre y otros mamíferos, parte superior y posterior de la cabeza, que comprende desde la frente hasta el cuello, excluida la cara.

Estas acepciones no presentan enmienda para la 23ª edición del DRAE.

(30) DRAE 2001 corazón. 1. m. Anat. Órgano de naturaleza muscular, común a todos los vertebrados y a muchos invertebrados, que actúa como impulsor de la sangre y que en el hombre está situado en la cavidad torácica.

Enmienda DRAE 23a ed. No presenta cambios en la acepción 1.

(31) DRAE 2001 diente. 1. m. Cuerpo duro que, engastado en las mandíbulas del hombre y de muchos animales, queda descubierto en parte, para servir como órgano de masticación o de defensa.

No presenta enmienda.

(32) DRAE 2001 humano, na. 1. adj. Perteneciente o relativo al hombre. 2. adj. Propio de él. 5. m. pl. Conjunto de todos los hombres.

Enmienda DRAE $23^{\mathrm{a}}$ ed. humano, na. 1. adj. Perteneciente o relativo al hombre (\| ser racional). No presenta cambios en las acepciones 2 y 5 .

(33) DRAE 2001 neolítico, ca. 1. adj. Se dice del último período de la Edad de Piedra, que supuso una revolución en muchos aspectos de la vida del hombre.

Enmienda DRAE 23a ed. neolítico, ca. 1. adj. Se dice del último período de la Edad de Piedra, caracterizado por sus innovaciones en el terreno de la técnica y de la organización económica y social.

(34) DRAE 2001 perro. 1. m. Mamífero doméstico de la familia de los Cánidos, de tamaño, forma y pelaje muy diversos, según las razas. Tiene olfato muy fino y es inteligente y muy leal al hombre.

No presenta enmienda.

(35) DRAE 2001 pulmón. 1. m. Órgano de la respiración del hombre y de los vertebrados que viven o pueden vivir fuera del agua.

No presenta enmienda.

(36) DRAE 2001 tórax. 1. m. Anat. Pecho del hombre y de los animales.

No presenta enmienda.

\subsection{Definición de estrategias semánticas parciales a partir del análisis del discurso lexicográfico del DRAE}

En primer lugar, hay que plantearse una cuestión inicial antes de abordar la definición de las estrategias semánticas presentes en el DRAE, y es el hecho de que las ideologías sean o no reconocidas conscientemente por todos los miembros que las comparten en una sociedad determinada. En este caso nos interesan los redactores y redactoras del discurso lexicográfico y gramatical académico, y la duda estriba en saber si son conscientes de la estrategia que definen y proyectan en el ámbito del sexismo o, por el contrario, si solamente responden a una automatización no controlada por la conciencia. Desde luego, en muchos casos no podremos hablar de la intencionalidad 
de determinadas formas y estructuras lingüísticas que aparecen en los discursos ideológicamente marcados, puesto que resultan herencia de una tradición cuya impronta es tan fuerte que queda imbricada con el conocimiento. No obstante, como advierte van Dijk (2005: 24) lo que cuenta no será el conocimiento consciente o la intención de los autores o autoras, sino cómo llega ese discurso y cómo puede ser interpretado.

Una vez asumido el hecho de que no resulta tan relevante la intencionalidad de la manifestación ideológica en el discurso como su interpretación posterior y la forma en la que afecta a determinados colectivos, podemos definir esas estrategias semánticas a las que hacíamos referencia. Con estrategias semánticas nos referiremos al conjunto de recursos que proyectarán un mensaje ideológico unitario. Puesto que nos ceñiremos al ámbito del sexismo lingüístico, hemos de entender estas estrategias como parciales, pues no remiten a la totalidad de la realidad sino a una parcela concreta de la misma. En concreto, sí se percibe una estrategia general, que podríamos enunciarcomominimización de la mujer, materializada no solo a través de la ausencia de pares morfológicos sino también del mantenimiento de acepciones que acusan obsolescencia. Por supuesto, dentro de esta estrategia se encontrarían otras más concretas y determinadas por una visión dicótoma hombre/mujer en la que a la mujer se le atribuirían de forma tácita y explícita características como la dependencia, la pasividad, la preponderancia de lo moral sobre lo intelectual, la transmisión frente a la creación, la diligencia en el ámbito familiar frente al profesional y la ausencia de capacidad de liderazgo. Desde luego, no podemos desarrollar todos los recursos de modalización discursiva que permiten este análisis, pero sí hemos de dejar constancia de que, de forma consciente o no, lo cierto es que el diccionario académico visibiliza indudablemente más al hombre que a la mujer. Un proceso análogo tendrá lugar también en las obras gramaticales, como demuestra sin ir más lejos un sencillo recuento y estudio de los ejemplos propuestos como forma de textualización en los manuales y gramáticas de la RAE y de otras editoriales que siguen un patrón semejante (Rodríguez Barcia 2012).

Por lo tanto, estas estrategias académicas inciden de manera directa en la forma de llegar a la lengua en el ámbito hispanohablante y condicionan sin lugar a dudas su modo de relación interpersonal a través del vehículo lingüístico. En este contexto, resulta clave pues la necesidad de llevar a cabo actuaciones concretas e intervenciones directas sobre el diccionario, pero también sobre la lengua empleada en diversos ámbitos, como el administrativo, el jurídico o, en general, en los ámbitos profesionales, pues efectivamente existen grupos de hablantes que se sienten minimizados y no representados en algunos usos que, no por tradicionales y presentes en las obras de la RAE, han de ser perpetuados.

\section{CONCLUSIONES}

La argumentación sobre el comportamiento del género gramatical en español justifica la pertinencia de la intervención en los usos lingüísticos con criterios de igualdad entre las personas, en particular para no perpetuar los efectos pragmáticos que se derivan en algunos casos del llamado masculino genérico.

Por otra parte, el análisis crítico del discurso lexicográfico de la RAE descubre la persistencia de una ideología todavía marcada por la minimización de la mujer, lo cual incide en la falta de visibilidad que es objeto de demanda social. Ideología y cultura pesan en los usos lingüísticos y es lícito por tanto compensar el androcentrismo dominante. 
Quedaría pendiente otro aspecto: en qué tipos de discurso es pertinente intervenir. Dejamos esta otra cuestión para futuras investigaciones.

\section{OBRAS CITADAS}

ADESSE. Base de datos de Verbos, Alternancias de Diátesis y Esquemas Sintáctico-Semánticos del Español. http://adesse.uvigo.es/data/ (Último acceso: 8 de marzo de 2013).

Aliaga García, Francisco y Fernando Lázaro Mora. 2003. “La 'marcación de género' en español” en José Luis Girón Alconchel (Ed.), Estudios ofrecidos al profesor José Jesús de Bustos Tovar. Madrid: Editorial Complutense. 5-22.

Ambadiang, Théophile. 1999. "La flexión nominal. Género y número" en Bosque, Ignacio \& Violeta Demonte (Dirs.), Gramática descriptiva de la lengua española 3. Entre la oración y el discurso. Morfología. Madrid: Espasa. 4843-4913.

Arias Barredo, Aníbal. 1990. "Género gramatical y motivación semántica". Estudios de Lingüística de la Universidad de Alicante 6: 107-121.

Bosque, Ignacio. 2012. "Sexismo lingüístico y visibilidad de la mujer". El País 4-3-2012: 14-17.

Buxó Rey, M. Jesús. 1978. Antropología de la mujer. Cognición, lengua e ideología cultural. Barcelona: Anthropos.

Bybee, Joan y Paul Hopper (Eds.). 2001. Frequency and the emergence of linguistic structure. Philadelfia: John Benjamins.

Cole, Michael. 1999. Psicología cultural. Madrid: Morata.

Corbett, Greville. 1991. Gender. New York: Cambridge University Press.

Corbett, Greville. 2011a. "Systems of gender assignment" en Dryer, Matthew S. \& Haspelmath, Martin (Eds.), The World Atlas of Language Structures Online. Munich: Max Plank Digital Library, feature 32A. Disponible en http://wals.info/feature/32A. (Último acceso: 8 de marzo de 2013)

Corbett, Greville. 2011b. "Sex-based and non-sex-based gender systems" en Dryer, Matthew S. \& Haspelmath, Martin (Eds.), The World Atlas of Language Structures Online. Munich: Max Plank Digital Library, chapter 31. Disponible en http://wals.info/chapter/31. (Último acceso: 8 de marzo de 2013)

Dixon, Robert M. W. 1982. Where have all the adjectives gone? Berlín: Walter de Gruyter.

Echaide, Ana Ma. 1969. "El género del sustantivo en español: evolución y estructura". Iberorromania 1: 89-124.

Forgas Berdet, Esther. 1996. "Lengua, sociedad y diccionario: La ideología." En Forgas Berdet, E. (Coord.), Léxico y diccionario. 71-90.

Forgas Berdet, Esther. 2006. "Un paso adelante: los fenómenos de la subjetividad lingüística en la última edición del diccionario académico". En Campos Souto, Mar y José Ignacio Pérez Pascual (Eds.), El Diccionario de la Real Academia Española: Ayer y Hoy, Anexos de Revista de Lexicografía 1: 69-83.

Forgas Berdet, Esther. 2007. "Diccionarios e ideología". Interlingüística 17: 2-16.

Forgas Berdet, Esther. 2011. "El compromiso académico y su reflejo en el DRAE: los sesgos ideológicos (sexismo, racismo, moralismo) del Diccionario" en Senz, Silvia y Montserrat Alberte (Eds.), El dardo en la Academia. Esencia y vigencia de las academias de la lengua española, Barcelona: Melusina. 425-457.

García Meseguer, Álvaro. 1994. ¿Es sexista la lengua española? Una investigación sobre el género gramatical. Barcelona: Paidós.

Greenberg, Joseph H. 1966. Language Universals. La Haya / París: Mouton.

Haspelmath, Martin. 2006. "Against markedness (and what to replace it with)". Journal of Linguistics 42: 25-70.

Jakobson, Roman. 1971. The selected writings of Roman Jakobson, vol. 2. La Haya: Mouton. Kerbrat-Orecchioni, Catherine. 1986. La enunciación. De la subjetividad en el lenguaje, Bue- 
nos Aires: Hachette.

Lakoff, George. 1987. Women, Fire, and Dangerous Things. Chicago / London: Chicago University Press.

Langacker, Ronald W. 1987. Foundations of Cognitive Grammar. Volume 1. Theoretical Prerequisites. Stanford, California: Stanford University Press.

Langacker, Ronald W. 2000. Grammar and Conceptualization. Berlín: Mouton de Gruyter.

Lledó, Eulàlia; M. Ángeles Calero Fernández y Esther Forgas Berdet (Coord.). 2004. De mujeres y diccionarios: evolución de lo femenino en la $22^{a}$ edición del DRAE. Madrid: Instituto de la Mujer.

Lledó, Eulàlia. 2012. A propósito del informe de la Real Academia Española de la Lengua (RAE): Que el bosque no te impida ver los árboles. Conferencia impartida en la Universidad Pública de Navarra, 21 de marzo de 2012. Disponible en: http://www.clasicasymodernas.org/2012/03/linguistica/conferencia-de-eulalia-lledo-sobre-el-informe-de-la-rae/ (Último acceso: 8 de marzo de 2013).

Moreno Cabrera, Juan Carlos. 2011. "Diversidad lingüística y diversidad cultural. Tipología, evolución y complejidad”, en J. J. Martos, L. Trapassi, I. García y V. M. Borrero (Eds.). Diálogos interculturales: lenguas, literaturas y sociedad. Barcelona: Anthropos. 11-41.

Moreno Cabrera, Juan Carlos. 2012. "Acerca de la discriminación de la mujer y de los lingüistas en la sociedad. Reflexiones críticas". Disponible en: http://es.scribd.com/ doc/85920261/Juan-Carlos-Moreno-Cabrera-UAM-\%C2\%AB\%E2\%80\%9CAcercade-la-discriminacion-de-la-mujer-y-de-los-linguistas-en-la-sociedad $\%$ E2\%80\%9D-Reflexiones-criticas\%C2\%BB (Último acceso: 2 de marzo de 2013).

Pardo Abril, Neyla Graciela. 2001. "Análisis del discurso: una lectura de la cultura" en Jaime Bernal Leongómez (Ed.), Lenguaje y cognición. Bogotá: Instituto Caro y Cuervo, 167-189.

Real Academia Española. 2001. Diccionario de la lengua española (22 ${ }^{a}$ edición). Madrid: Espasa-Calpe. Edición en línea: http://www.rae.es/rae.html (Último acceso: 8 de marzo de 2013).

Real Academia Española: Banco de datos (CREA) [en línea]. Corpus de referencia del español actual. http://corpus.rae.es/creanet.html (Último acceso: 8 de marzo de 2013).

Real Academia Española. 2001b. Nuevo Tesoro Lexicográfico de la Lengua Española. Madrid: Real Academia Española / Espasa Calpe. Edición en línea: http://buscon.rae.es/ntlle/SrvltGUISalirNtlle (Último acceso: 8 de marzo de 2013).

Real Academia Española / Asociación de Academias de la Lengua Española. 2009. Nueva gramática de la lengua española. Morfología. Sintaxis I. Madrid: Espasa.

Roca, Ignacio M. 2005. "La gramática y la biología en el género del español ( $1^{a}$ parte)”. Revista Española de Lingüística 35/1: 17-44.

Roca, Ignacio M. 2006. "La gramática y la biología en el género del español ( $2^{a}$ parte)”. Revista Española de Lingüística 35/2: 397-432.

Rodríguez Barcia, Susana. 2012. "El papel de los ejemplos en la construcción de estereotipos. Análisis del Compendio de la Gramática de la Lengua Española (Academia Española, 1931)”. Félix San Vicente y María Luisa Calero Vaquera (Eds.), Discurso de género y didáctica. Relato de una inquietud. Bologna: CLUEB. 153-173.

Trubetzkoy, Nikolaj. 1939. Grundzüge der Phonologie. Göttingen: Vandenhoeck \& Ruprecht. Trad. al español de Delia García Giordano con la colaboración de Luis J. Prieto. 1976. Principios de fonología. Madrid: Cincel.

UGT. 2008. Guía sindical de lenguaje no sexista. Madrid: Unión General de Trabajadores de Madrid, Secretaría de Igualdad.

van Dijk, Teun A. 1999. Ideología. Una aproximación multidisciplinaria. Barcelona: Gedisa editorial.

van Dijk, Teun A. 2005. "Ideología y análisis del discurso". Estudio. Utopía y Praxis Latinoamericana 29: 9-36. 
van Dijk, Teun A. 2010. "Noticias, discurso e ideología". Bahíllo Sphonix-Rust, Beatriz Burgos Cuadrillero y Ana María Iglesias Botrán (Coords. y Eds.), Interlingüística XXI. Actuales líneas de investigación en el panorama internacional de los jóvenes lingüistas. Valladolid: Universidad de Valladolid. 15-30.

Vygotsky, L. 1995. Pensamiento y lenguaje. Buenos Aires: Paidós. 
